

\title{
Synthesis of the Polymorphic Forms of Clopidogrel Hydrogen Sulfate Through Reactive Crystallization and Transformation
}

\author{
J. Lu* , L.W. ChEn, J. WANG and Z. Li
}

National Engineering Laboratory for Cereal Fermentation Technology, School of Chemical and Material Engineering, Jiangnan University, Wuxi, P.R. China

*Corresponding author: Fax: +86 510 85917763; Tel: +86 510 85919779; E-mail: dr.lujie@ gmail.com

(Received: 16 December 2011;

Accepted: 10 October 2012)

AJC-12265

\begin{abstract}
This work reports the preparation, solid state characterization and solvent-mediated transformation of the amorphous, I and II forms of clopidogrel hydrogen sulfate. In particular, labile precipitate and oiling out occurring in the reactive crystallization of clopidogrel hydrogen sulfate are firstly reported and analyzed. The occurrence domains of three forms are also schematically outlined as well. These results will contribute to a better understanding about the polymorphic nature of clopidogrel hydrogen sulfate, together with the approaches to synthesizing its pure forms.
\end{abstract}

Key Words: Clopidogrel hydrogen sulfate, Solid forms, Transformation, Reactive crystallization.

\section{INTRODUCTION}

Active pharmaceutical ingredients are frequently delivered to the patient in the solid state as part of such dosage forms as tablets, capsules, etc. ${ }^{1}$. No matter whether as pure drug substances or in formulated products, active pharmaceutical ingredients can exist in a great variety of distinct solid forms, such as polymorphs, pseudopolymorphs (solvates and hydrates), salts, co-crystals and amorphous solids ${ }^{2}$. Due to the differences in packing and/or conformation of the molecules, different solid forms usually exhibit different physical and chemical properties, corresponding to both clinical efficacy and dosage forms in the pharmaceutical industry ${ }^{3}$. Thus, in both theoretical and practical contexts, identifying the most appropriate solid form and controlling its crystallization is a key element in the developmental process for a new drug ${ }^{4}$.

Crystallization is not only in general the last chemical purification step in the production of active pharmaceutical ingredients but also an effective means to control their physical and chemical properties including crystal form, size and shape which have the potential to impact bioperformance ${ }^{5,6}$. There are a number of ways in which an active pharmaceutical ingredient can be crystallized from a solution, e.g., reaction, cooling, precipitation (drown out), evaporation, or a combination of these techniques ${ }^{7,8}$. Clopidogrel hydrogen sulfate (CHS) (CAS registry no. 120202-66-6, methyl (+)-(S)-a-(2chlorophenyl)-6,7-dihydrothieno[3,2-c]pyridine-5(4H) acetate, Fig. 1), a selective and irreversible inhibitor of adenosine-5'- diphosphate (ADP)-induced platelet aggregation, was widely used in the clinical treatment of atherosclerosis, acute coronary syndrome and other vascular diseases ${ }^{9}$. To date, though some works have been conducted to prepare its different forms, the exact crystallization mechanisms have not been clarified in detail $^{10}$.<smiles>CC(=O)[C@H](c1ccccc1Cl)[NH+]1CCc2sccc2C1</smiles>

Fig. 1. Chemical structure of clopidogrel hydrogen sulfate

In this work, reactive crystallization and transformation were used to prepare pure amorphous, I and II forms of clopidogrel hydrogen sulfate, followed by the solid state characterization using powder X-ray diffraction, Fourier transform infrared spectroscopy, differential scanning calorimetry and solubility measurement.

\section{EXPERIMENTAL}

Raw clopidogrel hydrogen sulfate of II form was supplied by Lixin Pharmaceutical Co. (Jiangsu, China). Ethyl acetate, dichloromethane, ethanol, sulfuric acid, sodium bicarbonate and anhydrous sodium sulfate, purchased from Sinopharm 
Chemical Reagent Co. (Shanghai, China), were of the highest grade available and used without further purification.

Preparation of three forms: The forms were prepared by use of reactive crystallization and solvent-mediated transformation respectively. During the reactive crystallization, the clopidogrel base was first prepared and dissolved into desired amount of ethyl acetate at room temperature. Under stirring sulfuric acid in a 1:1 mol ratio with the base was slowly dropped into the solution of base. The contents of solid forms were monitored by use of FTIR through interval sampling. When the content of desired form in suspension reached $99.5 \%$, the suspension was filtered off and then the product was washed and dried in a vacuum oven for $24 \mathrm{~h}$ at room temperature. Meanwhile, the oiling-out in the reaction crystallization was observed under an optical microscope and recorded.

Power XRD was conducted on a $\mathrm{D}_{8}$ advance X-ray diffractometer (Bruker AXS, Karlsruhe, Germany) at $40 \mathrm{kV}$ and $40 \mathrm{~mA}$ with a Ni-filtered $\mathrm{CuK}_{\alpha}$ radiation source $(\lambda=$ $1.5406 \AA)$. The samples were scanned from $5^{\circ}$ to $35^{\circ}(2 \theta)$ at a scanning rate of $0.5^{\circ}$ per min. The diffractograms were processed using the recent version of EVA software.

FTIR spectra were recorded from $\mathrm{KBr}$ disks using a Digilab Excalibur Series FTS-3000 spectrophotometer (Digilab, Canton, MA). Ground $\mathrm{KBr}$ powder was used. The measured wave number range was from 4000 to $500 \mathrm{~cm}^{-1}$. The construction of the calibration curves for quantitative analyses between amorphous form and I form and between I form and II form, were followed the work of $\mathrm{Lu}$ and Rohani ${ }^{11}$.

Differential scanning calorimetry was conducted by use of a Mettler-Toledo differential scanning calorimetry-822e differential scanning calorimeter operating with version 9.3 of Stare software (Mettler-Toledo, Columbus, OH). Accurately weighed samples (4-6 mg) were placed in hermetically sealed aluminum pans and scanned from $25^{\circ} \mathrm{C}$ to $200{ }^{\circ} \mathrm{C}$ at $10^{\circ} \mathrm{C} /$ min under nitrogen purge.

Solubility measurements: The solubility of three forms of clopidogrel hydrogen sulfate was measured in ethyl acetate at various temperatures. Saturated solution of each form was prepared in a $200-\mathrm{mL}$ jacketed glass crystallizer by adding excess powders of pure form. The temperature of the crystallizer was controlled by a F32 ME refrigerated circulator (Julabo Labortechnik, Seelbach, Germany) and the solution was stirred for $0.5 \mathrm{~h}$. Then the agitation was stopped and the suspension was allowed to settle for another $0.5 \mathrm{~h}$. The supernatant in equilibrium with a macroscopically observable solid was filtered through Millex-VV 0.1-mm filters (Millipore, Billerica, MA); and the solid residue was dried for FTIR analysis. The concentration of filtered supernatant was determined by use of the dry mass method. All experiments were replicated three times.

Solvent-mediated transformation: The solvent-mediated transformation from amorphous form to I form and that from I form to II form were studied respectively. Suspensions of the excess amount of pure amorphous form or I form in ethyl acetate ( $1 \mathrm{~g}$ solid in $27 \mathrm{~mL}$ ethyl acetate) were stirred in a $200-\mathrm{mL}$ jacketed glass crystallizer at $20,25,35$ and $40^{\circ} \mathrm{C}$. A portion of each suspension was withdrawn and filtered at designated times and the form composition in the solid phase was determined by FTIR.

\section{RESULTS AND DISCUSSION}

Fig. 2 shows typical powder XRD patterns of the three forms of clopidogrel hydrogen sulfate. For example, amorphous form has no peaks, while I form and II form have characteristic diffraction peaks at $9.2^{\circ}, 10.9^{\circ}, 11.6^{\circ}$ and $8.9^{\circ}, 9.8^{\circ}, 12.4^{\circ}, 13.0^{\circ}$, $13.8^{\circ}$, respectively.

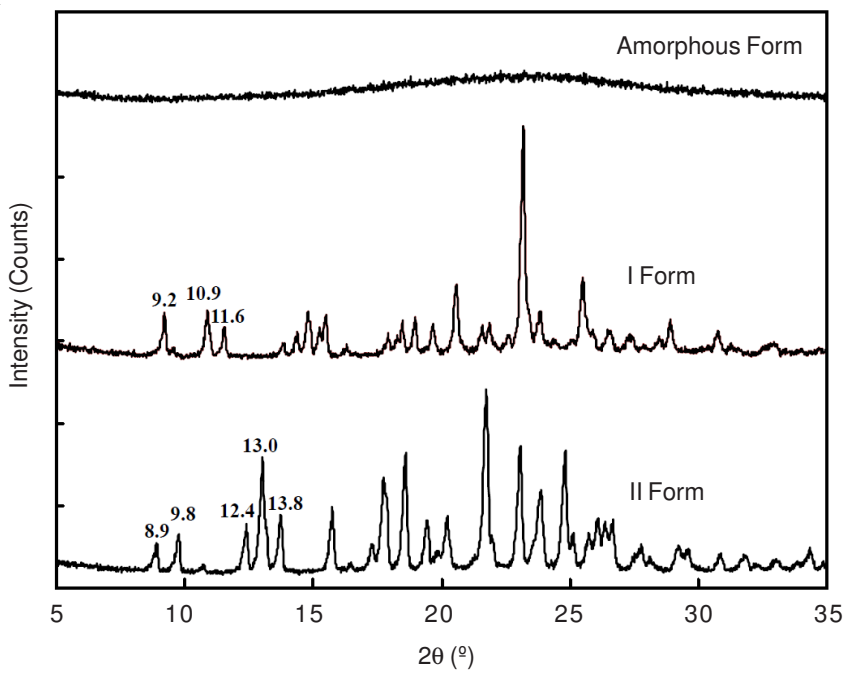

Fig. 2. Powder X-ray diffraction patterns of the three forms of clopidogrel hydrogen sulfate

The IR-spectra in the range of 1800 to $800 \mathrm{~cm}^{-1}$ of three forms are shown in Fig. 3. For instance, amorphous form, I form and II form have characteristic absorption bands at 1043, 1067 and $867 \mathrm{~cm}^{-1}$, respectively, while absorption band at 1752 $\mathrm{cm}^{-1}$ is common to all three forms. The differences in infrared spectra of three forms can be attributed to the difference in their spatial packing.

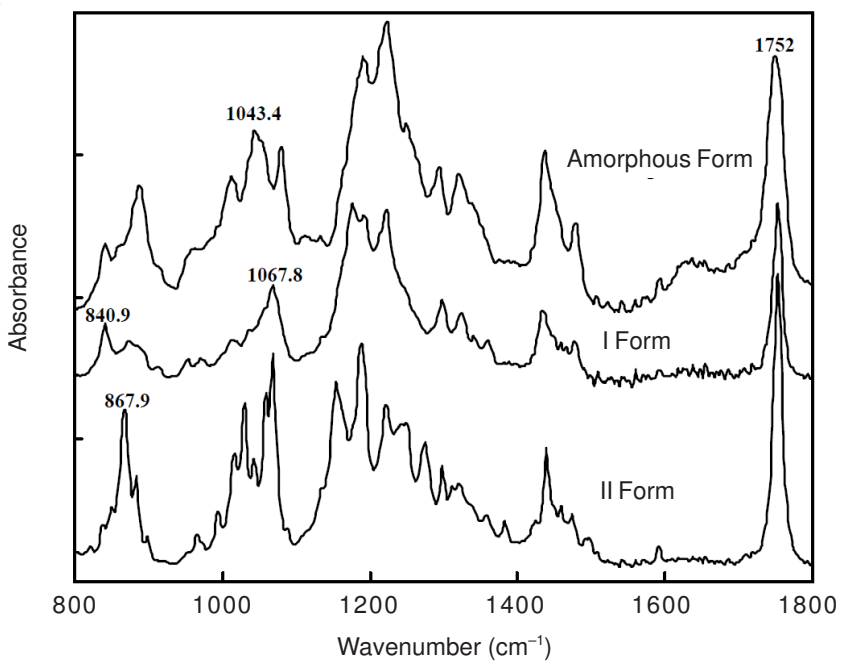

Fig. 3. FTIR spectra of the three forms of clopidogrel hydrogen sulfate

Typical differential scanning calorimetry thermograms of the three forms of clopidogrel hydrogen sulfate are shown in Fig. 4. Amorphous form has no specific melting endotherm with a glass transition temperature of $115.5^{\circ} \mathrm{C}$, while the measured onset and peak maximum of the melting endotherm of I form are $181.0^{\circ} \mathrm{C}$ and $186.2^{\circ} \mathrm{C}$, respectively and those of 


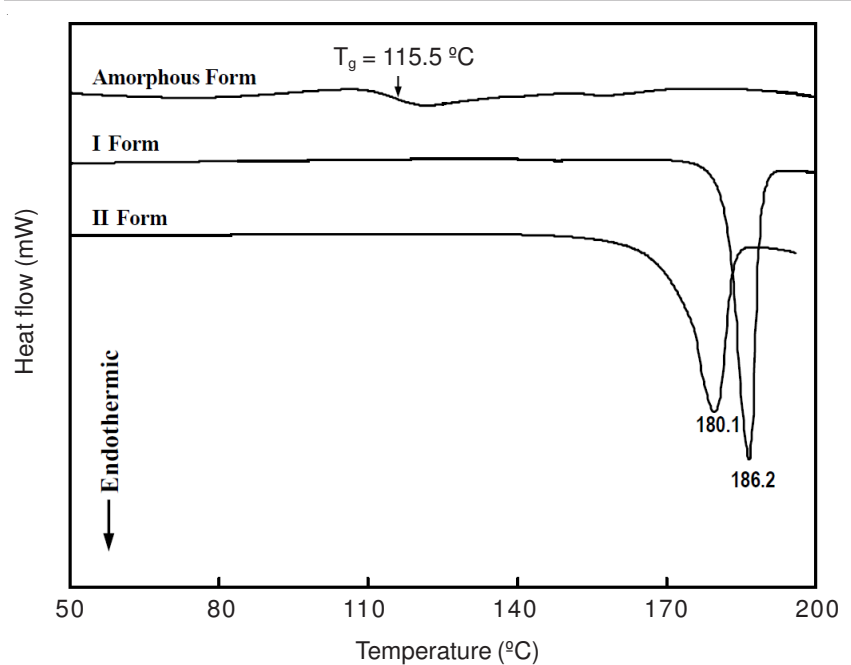

Fig. 4. Differential scanning calorimetry curves of the three forms of clopidogrel hydrogen sulfate

II form are $177.4^{\circ} \mathrm{C}$ and $180.1^{\circ} \mathrm{C}$, respectively. The measured melt enthalpy of forms I and II are $33.0 \mathrm{~kJ} / \mathrm{mol}$ and $35.0 \mathrm{~kJ} /$ mol, respectively.

Solubility and thermodynamic stability: Fig. 5 presents the solubility of the three forms of clopidogrel hydrogen sulfate in ethyl acetate at different temperatures, which in general increase with the temperature and decrease in the order: amorphous form $>$ I form $>$ II form.

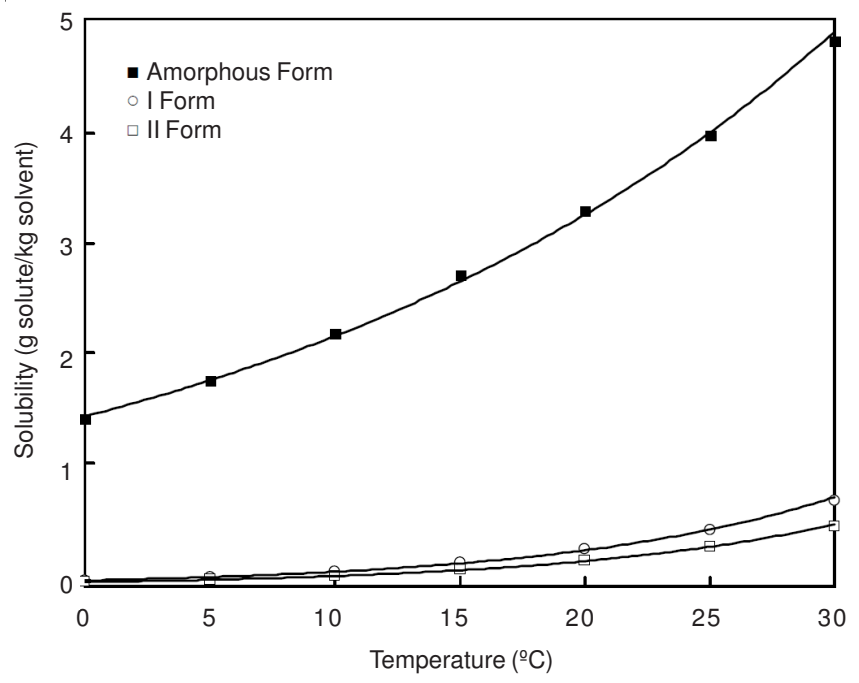

Fig. 5. Solubility of the amorphous, I and II forms of clopidogrel hydrogen sulfate in ethyl acetate at various temperatures

Predicted by heat-of-fusion rule and solubility rule ${ }^{12}$ amorphous form is the most thermodynamically unstable form, whereas the II form is the most thermodynamically stable form and amorphous and I forms are monotropically related while I and II forms are enantiotropically related.

Oiling out and occurrence domains: During the reactive crystallization of clopidogrel hydrogen sulfate, the firstly formed product was a kind of labile white precipitate (Fig. $6 a)$, which can quickly turn into a heavy viscous oil phase when it was withdrawn from mother liquor (Fig. 6b). This phenomenon has been termed as oiling out, liquid-liquid demixing or liquid-liquid phase separation (LLPS) ${ }^{13}$. The oil phase then stuck on the wall or the bottom of the crystallizer and when it was dried under vacuum at room temperature, a cake-like solid was obtained (Fig. 6c), which was confirmed to be amorphous by use of powder XRD.
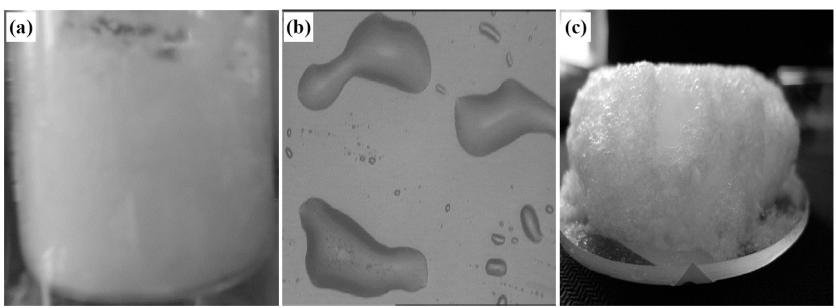

Fig. 6. Labile precipitate (a), oil droplets (b) and amorphous solid (c) of clopidogrel hydrogen sulfate

After monitoring and analyzing the process, the reactive crystallization of clopidogrel hydrogen sulfate can be schematically illustrated as Fig. 7a, in which all phases proceed in the following order: labile precipitate $\rightarrow$ oil phase $\rightarrow$ I form $\rightarrow$ II form. Accordingly their occurrence domains can be outlined in Fig. $7 b$.

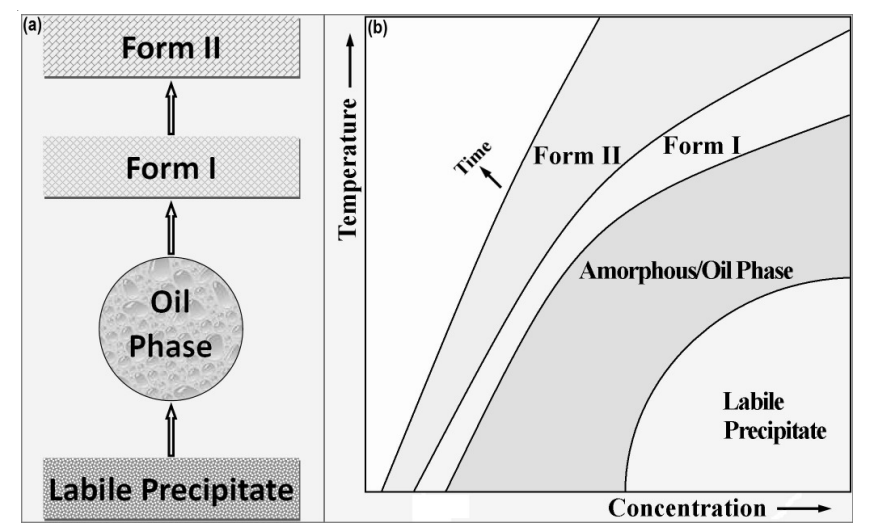

Fig. 7. Transformation order (a) and schematic occurrence domains (b) of the different phases of clopidogrel hydrogen sulfate during reactive crystallization

Solvent-mediated transformation: According to Fig. 7 , to prepare pure forms, the transformations between them should be determined. The calibration curves for quantitative analyses are shown in Fig. 8. The calibration curves plotted exhibit a good linearity over nearly the entire content range studied, which suggests that the simple approach applied in this work for the quantification of the polymorphic mixture of clopidogrel hydrogen sulfate via FTIR is practical ${ }^{14}$.

As shown in Figs. 9 and 10, the transformation from amorphous form to I form is much faster than that from I form to II form, which gives a narrow window to prepare pure forms separately. Both transformation rates remarkably increase with the temperature. This is because, increasing temperature will not only increase the nucleation rate constant, but also will remarkably increase the difference in solubility of two forms, thus results in enhanced transformation rates.

Eight kinetic models have been used to simulate the transformation processes ${ }^{15}$ (Table-1). Meanwhile, pre-exponential factors $\mathrm{k}_{0}$ and activation energies $\mathrm{E}$ of both reactions, calculated according to $\ln \mathrm{k}=-\mathrm{E} / \mathrm{RT}+\ln \mathrm{k}_{0}$, are given in Table-2. 


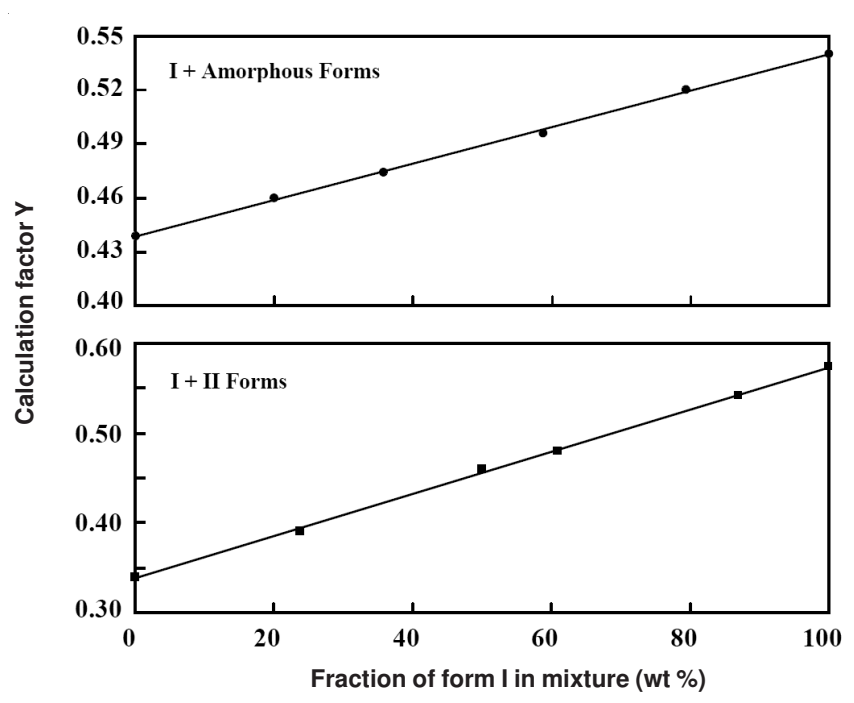

Fig. 8. Calibration curves for the quantitative analyses of the content of I form in the mixtures



Fig. 9. Mass fractions of form I in the transformation from amorphous form as a function of time, in ethyl acetate ( $1 \mathrm{~g}$ solid/27 $\mathrm{mL}$ solvent), at different temperatures

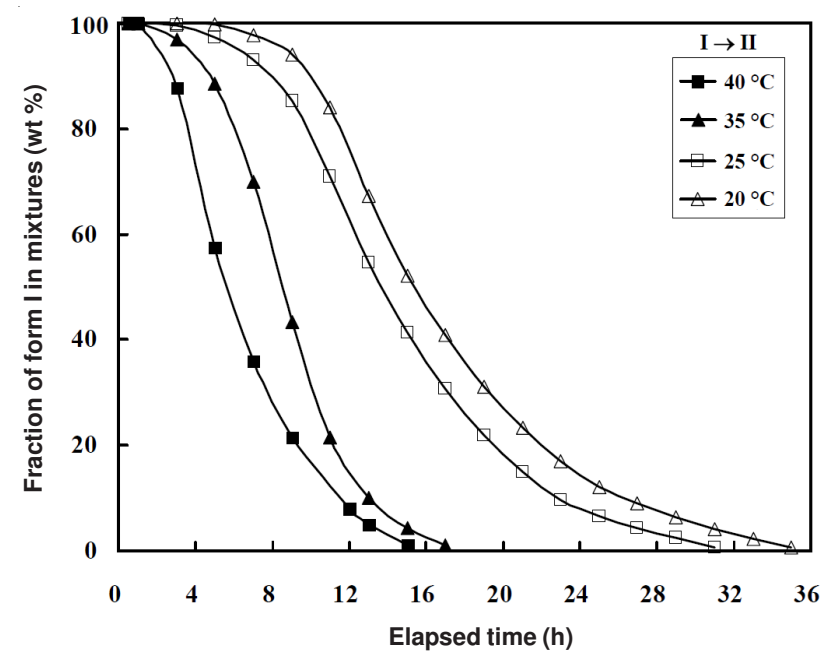

Fig. 10. Mass fractions of form I in the transformation to form II as a function of time, in ethyl acetate ( $1 \mathrm{~g}$ solid/27 $\mathrm{mL}$ solvent), at different temperatures
TABLE-1

KINETIC MODELS FOR SIMULATING THE TRANSFORMATION REACTIONS

\begin{tabular}{|c|c|c|c|}
\hline Kinetic models & \multicolumn{3}{|c|}{ Equations $\mathrm{a}, \mathrm{b}, \mathrm{c}$} \\
\hline Prout-Tompkins & \multicolumn{3}{|c|}{$\ln [\alpha /(1-\alpha)=k t+c$} \\
\hline Avrami-Erofeev & \multicolumn{3}{|c|}{$[-\ln (1-\alpha)]^{1 / n}=k t(n=1,2,3)$} \\
\hline One dimensional phase boundary & \multicolumn{3}{|c|}{$1-\alpha=\mathrm{kt}$} \\
\hline Two dimensional phase boundary & \multicolumn{3}{|c|}{$1-(1-\alpha)^{1 / 2}=\mathrm{kt}$} \\
\hline Three dimensional phase boundary & \multicolumn{3}{|c|}{$1-(1-\alpha)^{1 / 3}=\mathrm{kt}$} \\
\hline One dimensional diffusion & \multicolumn{3}{|c|}{$\alpha^{2}=\mathrm{kt}$} \\
\hline Two dimensional diffusion & \multicolumn{3}{|c|}{$(1-\alpha) \ln (1-\alpha)+\alpha=\mathrm{kt}$} \\
\hline Three dimensional diffusion & \multicolumn{3}{|c|}{$\left[1-(1-\alpha)^{1 / 3}\right]^{2}=\mathrm{kt}$} \\
\hline \multicolumn{4}{|c|}{$\begin{array}{l}{ }^{\mathrm{a}} \mathrm{k} \text { is the reaction rate constant; }{ }^{\mathrm{b}} \alpha \text { is the fraction transformed; }{ }^{\mathrm{c}} \mathrm{t} \text { is the } \\
\text { reaction time }\end{array}$} \\
\hline \multicolumn{4}{|c|}{$\begin{array}{c}\text { TABLE-2 } \\
\text { KINETIC MODELS APPLICABLE TO THE TRANSFORMATION } \\
\text { PROCESSES OF CHS SOLID FORMS }\end{array}$} \\
\hline Equations & $\begin{array}{c}\mathrm{k}_{0} \times \\
10^{-4}\end{array}$ & $\begin{array}{c}\mathrm{E} \\
(\mathrm{kJ} / \mathrm{mol})\end{array}$ & $\begin{array}{l}\text { Jorrelatior } \\
\text { :oefficient: } \\
\quad\left(\mathrm{r}^{2}\right)\end{array}$ \\
\hline$[-\ln (1-\alpha)]^{1 / 2}=\mathrm{kt}$ & 4241 & 43.52 & 0.9792 \\
\hline$[-\ln (1-\alpha)]^{1 / 3}=\mathrm{kt}$ & 4.480 & 33.47 & 0.9794 \\
\hline
\end{tabular}

\section{Conclusion}

In this work the amorphous, I and II forms of clopidogrel hydrogen sulfate were prepared through reactive crystallization and solvent-mediated transformation and were characterized by use of PXRD, FTIR and differential scanning calorimetry.

\section{ACKNOWLEDGEMENTS}

This work was supported by the National Natural Science Foundation of China (Nos. 21176102 and 21176215), the Scientific Research Foundation for Returned Chinese Scholars (Yr 2011) and the Fundamental Research Funds for the Central Universities (No. JUSRP30904).

\section{REFERENCES}

1. S.L. Morissette, Ö. Almarsson, M.L. Peterson, J.F. Remenar, M.J. Read, A.V. Lemmo, S. Ellis, M.J. Cima and C.R. Gardner, Adv. Drug Deliv. Rev., 56, 275 (2004).

2. S.R. Byrn, R.R. Pfeiffer and J.G. Stowell, Solid State Chemistry of Drugs, SSCI Inc., West Lafayette, p. 16 (1999).

3. J.K. Haleblian and W.J. McCrone, J. Pharm. Sci., 58, 911 (1969).

4. J. Lu and S. Rohani, Org. Proc. Res. Dev., 13, 1262 (2009).

5. A.H. Mangood, M.M. Seif and S.M. Hamza, Asian J. Chem., 22, 7257 (2010).

6. W. Yue and G. Nie, Asian J. Chem., 21, 6295 (2009).

7. M.V. Krishna, J.R. Babu, P.V.M. Latha and D.G. Sankar, Asian J. Chem., 19, 1369 (2007).

8. J. Ahmad, Asian J. Chem., 14, 223 (2002).

9. J.S. Dickie and L.J. Scott, Am. J. Cardiovasc. Drug, 6, 407 (2006).

10. H.J. Kim and K.J. Kim, Ind. Eng. Chem. Res., 48, 11133 (2009).

11. J. Lu and S. Rohani, Curr. Med. Chem., 16, 884 (2009).

12. J. Bernstein, R.J. Davey and J.O. Henck, Angew. Chem. Int. Ed., 38, 3440 (1999).

13. L. Codan, M.U. Bäbler and M. Mazzotti, Cryst. Growth Des., 10, 4005 (2010).

14. P.J. Skrdla, V. Antonucci, L.S. Crocker, R.M. Wenslow, L. Wright and G. Zhou, J. Pharm. Biomed. Anal., 25, 731 (2001).

15. L.E. O'Brien, P. Timmins, A.C. Williams and P. York, J. Pharm. Biomed. Anal., 36, 335 (2004). 